\title{
AN ANALYSIS OF REQUIREMENTS, OPPORTUNITIES, AND CHALLENGES OF WIRELESS NETWORK VIRTUALIZATION
}

\begin{tabular}{|c|c|c|c|}
\hline \multicolumn{4}{|c|}{$\begin{array}{l}\text { Swapnil Raj } \\
\text { ersity, Mathura, Uttar Pradesh, India }\end{array}$} \\
\hline Journal & \multicolumn{3}{|c|}{$\begin{array}{l}\text { Samvakti Journal of Research in Information Technology } \\
\text { https://www.sjrit.samvaktijournals.com } \\
\text { Volume } 2 \text { Year of Volume } 2021 \text { Page No : } 157 \text { - } 164\end{array}$} \\
\hline Discipline & \multicolumn{3}{|l|}{ Networking } \\
\hline Conference & \multicolumn{3}{|c|}{$\begin{array}{l}\text { A virtual international conference on redefining and transforming the role of higher } \\
\text { education in sustainable development }\end{array}$} \\
\hline Conference & \multicolumn{3}{|c|}{ Start Date: September 30, 2021} \\
\hline Dates & \multicolumn{3}{|c|}{ End Date : September 30, 2021} \\
\hline Institute Name & \multicolumn{3}{|c|}{$\begin{array}{l}\text { JAIN (Deemed-to-be University) in association with Council for Industrial } \\
\text { Innovation and Research }\end{array}$} \\
\hline $\begin{array}{l}\text { Date Received } \\
\text { ID } \\
\text { Dol }\end{array}$ & $\begin{array}{l}: \text { November } 21,2021 \\
: 2021.02 .29 \\
: 10.46402 / 2021.02 .29\end{array}$ & $\begin{array}{l}\text { Publication Date } \\
\text { Paper Type } \\
\text { Dol URL }\end{array}$ & $\begin{array}{l}\text { : December 16, } 2021 \\
: \text { Conference Paper } \\
: \text { https://dx.doi.org/10.46402/2021.02.29 }\end{array}$ \\
\hline
\end{tabular}

\section{ABSTRACT}

Wireless network virtualization (WNV) has piqued interest of academic and industrial researchers as one of most important technology in cellular network communication. With de coupling networks function of co-occurring virtual network, it is regarded as a pioneer in achieving efficient resource use with reduced operational and capital costs. It allows for the rapid arrangement of novel service \& technology. The WNV model is still in its initial phases, therefore here is plenty of opportunity with new architectures, systems, and applications to be developed by the research community. The accessibility of softwaredefined networking (SDN) \& cloud/centralized radio access network (C-RAN) raises the possibility of WNV becoming a reality. The article examines WNV, as well as current advances in SDN and C-RAN technology. We recognise needs \& possibilities of upcoming cellular system depending on these technology and WNV ideas. Then, based on SDN, we suggest a generic architectural framework for the WNV. There will also be a detailed discussion of the difficulties \& investigation concerns, alongwith potential methods for upcoming network communication enhancements. Finally, we provide a number of potential possibilities for upcoming network service for both domestic \& commercial users. 
KEYWORDS: Data, Mobile Network Operators, Software-Defined Networks, Virtualization, Wireless.

\section{INTRODUCTION}

The rise of wireless data traffic and services has been phenomenal for mobile network operators (MNOs), and this trend is likely to continue in the coming years. The flow of data traffic would exceed the zettabyte (ZB) barrier in 2016, according to a Cisco study, hitting 88.7 exabytes (EB) each month. According to study, flow of traffic would stretch to 2.3 ZB by 2020 . Internet, Mobile and smart device is main driver, resulting in enlarged video traffic, real-time gaming, \& web-based application service. With result of such growth, system administration \& configuration become more complicated, requiring MNOs to raise both capital and operational costs (OPEX). Regrettably, the increase of data traffic demand is inversely proportional to revenue growth. MNOs have a difficult and expensive task in deploying wireless network infrastructure to accommodate this pace of development. Because of the system's complexity, growing no. of base stations (BSs) to aim for boosting capacity of network wasn't ideal option. As a result, MNOs have agreed to share resources as a major way of lowering new deployment and operating costs. Software-defined networks (SDN), wireless network virtualization (WNV), and cloud radio access networks (C-RAN) have all emerged as potential technical solutions to this problem. These technologies make efficient system sharing of resource \& versatile arranging easier to deploy. SDN allows control \& data plane to be separated, increasing system flexibility and scalability. Networks become programmable, centrally controlled, flexible, and cost efficient as a result of this strategy. WNV allows for the separation of network functions from physical networks, allowing for faster network resource sharing.

Apart from resource sharing, WNV makes it simpler to migrate to novel technology whereas still maintaining older items by segregating a portion of the network. For developing heterogeneous wireless networks settings, SDN \& WNV offer connected and authoritative management method of network. The remote radio head $(R R H)$ of a conventional BS is separated from the baseband unit (BBU) by C-RAN. Through highspeed fronthaul, hundreds RRHs may linked to sole BBU (usually the fiber optics). As per novel RAN design, C-RAN may address a number of issues that now plague third- and 4th-generation (3G/4G) network, for instance intrusion \& high power depletion. C-RAN could support 5th generation (5G) technology like large scale antenna systems, fullduplex, and, ultra-dense networks because to flexibility \& scalability of execution which is based on cloud \& its inherent centralized nature. It enables many heterogeneous networks to share spectrum and BBU resources. As virtual BS was housed in centralized $\mathrm{BBU}$, changeover in mobile VNs is simplified. As a result, the 3 technology functioning simultaneously may provide greatest performance to upcoming system of network since every technology have unique feature that complements the others. This necessitates a new architectural design to support and assist future network system modifications and demands[1]. 
The NFV idea has resulted in WNV. NFV is a powerful new technology that explains the idea of executing network operations that were previously performed on a dedicated network hardware appliance in form of application software in generic server architecture. Virtualization layer virtualizes actual hardware resources such as compute, storage, and network in NFV system (e.g. hypervisor). Traditional network functions (example, load balancers, firewalls) are transformed in software application (Virtualized network Functions-(VNF)) \& operate in general-purpose hardware using NFV. It takes use of information technology environment's high processing capabilities to build more flexible network settings. Commercial-off-the-shelf (COTS) computer gear is used instead of custom-built hardware.

\section{Sharing of infrastructure in WNV:}

Infrastructures are support system of every network system which is wireless. They are expensive to purchase and maintain. In today's context, MNOs own all or portions of the infrastructure. As previously said, given the present trend, infrastructure sharing is unavoidable for dropping CAPEX \& OPEX however enhancing \& delivering high-quality service to consumers. Network infrastructure sharing, according to, refers to a situation where several network use identical external network structures on approved-upon terms \& condition. Radio spectrum \& components of the access network is two resource which may distributed. As a result, external sharing of network may be stated as radio spectrum distribution, wireless network infrastructure sharing, or both. The radio frequency have developed in white spectrum (hole) as a result of the availability of cognitive radio, which are the underutilized spectrum by the owner at any given moment. Other users may use this on condition that it don't conflict with user which is licensed. Infrastructure distribution may take place in two ways: passively or actively. Passive sharing refers to distribution of component which are non-network like premises, whereas active sharing discusses distribution of network parts like routers, eNBs, backbone transmission, backhaul and so on. Sharing may either be supportive or antagonistic. Non-cooperative sharing may be used for materials created for the general public. Cooperative sharing must be established if this is not the case. In the 3GPP standard, spectrum and infrastructure sharing are combined to create complete network sharing, which is enabled by two architectural configurations: multi-operator core network (MOCN) and core gateway network (CGWN). Former entails distribution of RANs, containing radio resources, whereas latter extends outside RAN distributing to include certain fundamental network components such as MSCs and SGSNs[2].

\section{Software Defined Network:}

SDN in $5 G$ assures to offer \& deploy novel skills \& resolutions intended for programmable, centrally controlled, adaptive, and cost-effective network management, making it ideal for high-bandwidth applications like video streaming. With reality, in SDN, the underlying network architecture is completely separated from applications whereas networking intelligence is logically controlled centrally. Various standardization groups and organizations, including the Open Networking Foundation (ONF), the Internet Research 
Task Force (IRTFSoftware )'s Defined Networking Research Group (SDNRG), and the Internet Engineering Task Force (IETF), are functioning on SDN standardization efforts. Describing SDN architectural constituents \& interface, specifying SDN \& manage capability for network which are wireless \& mobile with use of OpenFlow, classifying SDN model \& taxonomy, defining serviceable requirement, \& forecasting SDN for future Internet are examples of standardisation activitiy[3].

\section{a. Advantages:}

- To Provide granular network traffic management and excellent enactment across a variety of network manufacturers' devices. Network managers may use network control to implement various QoE/QoS rules at the application, network, and session device levels.

- Centralized network control and administration, which improves automation and makes corporate provisioning and management simpler to centralize.

- Providing a better end-user experience via network apps that can utilize centralized network intelligence \& state data for smoothly adapt to network circumstances and behaviors based on the requirements of the users.

- Improving network versatility through enabling novel service \& capabilities of network without requiring individual device configuration.

- Improving network stability and security by using autonomic network device management and policy enforcement consistency.

- Providing operators, businesses, users, and independent software suppliers with improved programmability opportunities/features/automation and network control through a common programming environment[4].

\section{C-RAN:}

C-RAN developed since conventional dispersed BSs, which included processing unit \& radio. These BSs' processing capability is dedicated to their mobile customers and is not shared over a wide geographic region. This is true, for example, in the $4 \mathrm{G}$ design, where inter-BS coordination is done through the X2 interface. BSs in commercial districts are overburdened throughout the day, while those in residential areas are inactive but nevertheless use a considerable amount of electricity, and the opposite. The necessity to upgrade existing RAN design to improved one that could reduce or eliminate current issue and release the capacity of these technologies has grown compelling as data traffic needs have increased. For addressing this issue, the C-centralized RAN's design was implemented[5].

\section{Challenges and Research Issues:}

Despite the fact that SDN and WNV have many beneficial characteristics in contemporary networks, there are still a number of major issues that are needed to be. Many academics have shown interest in and confidence in these pioneer technologies in order to meet the increasing consumer request meant for upcoming network system. Advent of C-RAN technology has increased people's faith in technology. There is multiple stages for 
apparatus of user to interact with another UEs, including setup of session among destination and source, as well as physical resource allocation to meet the need. Additionally, in the data forwarding process, efficient and flexible management of resource should provide QoE and offer better performance of system. In this part, we discuss some of the difficulties that must be solved in order for future networks systems to meet client demand.

\section{a. System Security:}

The security risks live in a dense ecosystem with intelligent gadgets from many technologies. WNV encourages various networks to pool their resources for providing better facility to the customers. SDN allows centralized systems to be controlled, but security measures must be properly-designed to guarantee the secrecy and concealment of user's data. Even with conventional wireless systems, security remains a challenge; for instance, smart phones have become a focus factor for cyber criminals. In a diverse context, the expansion and accessibility of content-aware and intellectual devices introduces additional risks beyond those seen in conventional wireless networks. To guarantee the system's integrity, secrecy, and non-repudiation, it needs a strong authentication mechanism. Because all systems have certain weak spots, multi-level security is required. DDoS attacks are one of the most common security concerns, particularly in resource sharing environments. In comparison to conventional networks, security risks are rapidly growing due to the de coupling of the control from the data plane. There are a number of security risks associated with SDN[6].

\section{b. Interference and Fading Channel:}

In wireless and wired settings, the transmission pathways are totally different. When related to wireless transmission media, the transmission medium in a wired network are directed, extremely dependable, and virtually have a consistent quality. However, since wireless channels have a broadcast characteristic, communiqué between 2 nodes influences the communication of rest of nodes, and signal propagation suffers random fading. Isolation is a necessary feature to allow the resources sharing and abstraction across various tenants in order for backing compact devices in similar infrastructures lacking interfering. It should be emphasized that the wishes are that any changes to virtual wireless networks' setup, customisation, or topology do not impact or interfere with coexisting virtual networks. For supporting this, they suggested a combination of timespace and source distribution system for WNV for improving seclusion through various trials, however their arrangement was computationally difficult owing to the high number of variables, some of which were manually modified[7].

\section{c. Interfacing:}

In wired virtualization, the requirements for virtual nodes and virtual links may be stated using a standard specific language. In WNV, SPs need resources related to radio from 1 or more InPs; however, the SPs may utilize distinct RATs while utilizing the same InP. 
Dissimilar to conventional wireless networks, where users are linked to a single MNO and are likely to employ the same RATs all the time. For InPs to comprehend radio needs of SPs, a standard interface is needed which should be well-defined. In order for VNs to be implemented, a ordinary language is needed to clearly describe the exchange of information across various InPs. Finally, in order to offer open standard interfaces, the proprietary dependency needs be resolved.

\section{d. Nodes Mobility:}

The unrestricted mobility of nodes and services is one among most significant characteristics of environment of mobile wireless network. One of benefits of wireless network systems, especially cellular networks, is this. However, in WNV, this advantage creates certain difficulties. The mobility management faces two additional difficulties as a result of this mobility. Due to the fact that the user could execute a position update through various MNVOs or handoff, latency and InPs issues arise. It differs from conventional wireless networks, in which the user is assigned to a single MNO and location updates are performed by a single operator. It must be obvious that handover and location of user are interdependent. As a result, a mobility management system is required to guarantee the monitoring and organisation of updates of location of all the users in all scenarios, including those with extremely high movement (example, trains with high speed, aircraft) and those with little to no movement. A qualitative mobility management system with effective allocation of resources is needed to optimize users' QoE in an environment which is dynamic.

\section{e. Network Management:}

Management is required to ensure the correct operation of host virtual wireless network, the physical infrastructures, and virtual networks' wireless services. Virtual network might span numerous underlying physical systems, posing new system managing problems. Instability in the state of user may cause SPs to alter the request of resources dynamically. As a result, there should be explicit network management procedures in place to prevent conflicts among the system's participants. Furthermore, the core network may be diverse, with distinct components having different unique characteristics, necessitating the development of unique solutions and methods for controlling WNV.

\section{DISCUSSION}

One of the most prominent developments amongst networked material \& communication technology (ICT) system is virtualization of network which is wired \& end computer system. In contrast, infrastructure-based wireless networks have seen very little virtualization, although the concept of virtualizing access is increasing traction since it have potency for enhancing spectrum use and possibly generate novel service. In the article, author review present status of wireless network virtualization investigation. We identify and explain potential designs, as well as problems, roadblocks, and trends in wireless network virtualization implementation. 


\section{CONCLUSION}

Because of the present rise in customer demand for real-time experiences, MNOs are looking for innovative methods to provide services at a reduced cost. WNV has arisen as a potential method for building network equipment much more open, allowing MNOs to be stretchier and adjust to new technologies more quickly. WNV may lower both CAPEX and OPEX by pooling network resources. WNV, when combined with closely related disciplines of SDN and C-RAN, offers communication systems optimistic future. We presented WNV, discussed its characteristics and contributions to future systems, and proposed an architectural framework built on SDN in this article. Many communication stakeholders have looked at WNV in form of solution for their present and future network problems because of its litheness, interoperability, unified management, arrangement, and service mechanization. However, there are currently no properly-defined standard of WNV. As a result, it is critical \& essential for relevant authorities to provide specifications and standards in order to promote new developments. Future cellular network technologies' needs and possibilities are highlighted. The ideas of SDN and C-RAN, as well as their characteristics, relationships through WNV, \& structural design, has thoroughly explored. Difficulties \& research concerns which would critical to WNV's achievement and that must be solved in order to bridge the gaps for future network communication enhancements are also discussed. 


\section{REFERENCES:}

[1] Q. Shi, L. Zhao, Y. Zhang, G. Zheng, F. R. Yu, and H. H. Chen, "Energy-Efficiency Versus Delay Tradeoff in Wireless Networks Virtualization," IEEE Trans. Veh. Technol., 2018, doi: 10.1109/TVT.2017.2738024.

[2] J. Van De Belt, H. Ahmadi, and L. E. Doyle, "Defining and Surveying Wireless Link Virtualization and Wireless Network Virtualization," IEEE Commun. Surv. Tutorials, 2017, doi: 10.1109/COMST.2017.2704899.

[3] F. Fu and U. C. Kozat, "Stochastic game for wireless network virtualization," IEEE/ACM Trans. Netw., 2013, doi: 10.1109/TNET.2012.2190419.

[4] X. Wang, P. Krishnamurthy, and D. Tipper, "Wireless network virtualization," J. Commun., 2013, doi: 10.12720/jcm.8.5.337-344.

[5] X. Lu, K. Yang, Y. Liu, D. Zhou, and S. Liu, "An elastic resource allocation algorithm enabling wireless network virtualization," Wirel. Commun. Mob. Comput., 2015, doi: 10.1002/wcm.2342.

[6] Z. Feng, C. Qiu, Z. Feng, Z. Wei, W. Li, and P. Zhang, "An effective approach to 5G: Wireless network virtualization," 2015, doi: 10.1109/MCOM.2015.7355585.

[7] X. Lu, K. Yang, and H. Zhang, "An elastic sub-carrier and power allocation algorithm enabling wireless network virtualization," Wirel. Pers. Commun., 2014, doi: 10.1007/s11277-013-1439-7.

\section{$\underline{\underline{E N D}}$}

